\title{
Index of places Part I
}

The index covers the main text, excluding the front matter, tables and bibliography. Names of places or institutions cited as the source of an image or a reference have not been included, nor are place names that are part of the title of artworks; neither is the Voortrekker Monument, which is omnipresent. The names of provinces that were current at the time have been added to South African towns for ease of identification. More search options are provided by the open access book.

\author{
Aliwal North (Eastern Cape) 72, 74 \\ Athens (Greece) \\ Parthenon 107, 136, 137, 146 \\ Bassae (Greece) \\ Temple of Apollo 137, 146, 242 \\ Beaufort West (Western Cape) 74 \\ Bellville (Western Cape) 74 \\ Berlin (Germany) 131, 132 n 524, 181, 185, 304, 306, \\ 323 \\ Damenakademie des Vereins Berliner \\ Künstlerinnen 304 \\ Great Altar from Pergamon 107, 109, 133, 242
}

Bethlehem (OFS) 67

Black Mfolozi River 384

Black Mountain (see also Thaba Nchu) 359

Bloemfontein (OFS) $31 \mathrm{n}$ 97, 43, 52, 61, 63, 74, 184, 185,

386, 387, 406

Archive for Contemporary Affairs (ARCA) 5, 6, 124,

154, 158, 160, 161, 167, 192

Grey College 184

Oranje Girls School (Christelike en Nasjonale

Meisieskool Oranje) 185, 186

Reserve Bank 117

Vrouemonument $27 \mathrm{n} 75,29,30,31,80,82,91$,

94, 95, 97, 107, 109, 116, 117, 119, 179 n 656, 261

Blood River (Ncome River) 5, 7, 10, 11, 13, 23, 24, 26, 27, $28,29,31,32,41,43,56,61,62,63,65$ n 288, 66 n $292,74,75,83,95,98,100,119,122,123,137$, 138, 139, 150, 152, 170 n 630, 181 n 668, 184 n 680, 201, 207, 208, 279, 282, 283, 299, 355, 367, 370, $371,375,377,379,383,384,395,405,409,435$, 436, 448, 450

Blood River Monument 6, 31 n 92, 50, 119 n 474, $125 \mathrm{n} 492$

Blood River Museum 236

Bloukrans (Natal) 24, 33 n 111, 62, 74, 120, 122, 123, 137, 166, 170 n 630, 297, 369, 370, 371, 375, 382, 436 n 1257

Bloukrans Monument 41, 87 n 377

Blydevooruitsig (see Kerkenberg)

Brighton (UK)

War Memorial 132

Brussels (Belgium) 305

Bushman's River 382, 383

Cambridge (UK), University 48

Cape (province) 36, 37, 48, 72, 74, 75, 88, 397, 407 n 1168

Victor Verster prison (Mandela statue) 416
Cape/Cape Colony 18, 19, 22, 25, 26, 27, 28, 31 n 94, $72,89,111,123,126,150,203,294,353,358,385$, $386,388,395,426,448$

Cape of Good Hope 21, 174

Cape Town 67 n 295, 72, 74, 75, 141, 181, 182 n 671 , 282, 397, 416 n 1200, 458

Castle 111, 434

Cecil Rhodes sculpture (UCT) 418

City Hall 228 n 803

House of Assembly 44

K club 181, 182

Louis Botha statue 262, 263, 268, 418, 419

Martin Melk House 182

Michael Stevenson galleries 458

National Library 7, 21, 369 n 1096

Old Mutual building and frieze 110-116, 137, 242,

437

University of Cape Town (UCT) 7, 48, 418

Carrara (Italy) 258, 259

Chieveley 41, 87 n 377

China 423

Cullinan (Transvaal)

Ditsong Willem Prinsloo Agricultural

Museum 407 n 1175

Danskraal (Natal) 61, 62, 63, 123, 125

Delagoa Bay (Maputo/Lourenço Marques, Mozambique) 19, 22, 27 n 70, 56, 124, 174, 206, 295, 357

Den Haag (The Hague, Netherlands) National Archives 6

Drakensberg Mountains 19, 22, 23, 25, 120, 123, 126, 151, 174, 294, 296, 299, 300, 357, 362, 365, 369, $375,384,385,386,387,431,436,439,443$

Dundee (Natal) Talana Memorial 49, 57 n 249, 87 n 377, 89 n 387

Durban (see also Port Natal) 22, 23, 258, 268, 275 n 933

University of Technology 413

East London (Eastern Cape)

Steve Biko statue 413

Edinburgh (UK)

Scottish War Memorial 133, 136, 137 n 535

Estcourt (Natal) 372, 375

Florence (Italy) 9, 177n 646, 252, 258, 303, 307 n 1007, 333

Accademia di Belli Arti 331, 332 
Fonderia Artistica in Bronzo ed Altri Metalli (Vignali foundry) 261, 262, 263

Romanelli studio $6,13,113,239,240,243,248$, 259, 263, 265-269, 282, 330, 332, 334, 336, 340, 352

Forte dei Marmi (Italy) 258 n 855, 259, 267, 345

Frankfurt am Main (Germany)

Heinrich Heine memorial 319,320

Geneva (Switzerland)

Monument de la Réformation 133

Germany 38, 132 n 524, 133, 181, 185, 188 n 699, 304, 305, 306 n 1006, 394, 423

Göttingen (Germany) 181

Graaff-Reinet (Eastern Cape) 72, 74, 353

Grahamstown (Eastern Cape) 74, 111, 124, 223, 284, 294, 353, 355, 358, 371

Bible Monument 436, 437

Masonic Lodge 353

Great Zimbabwe 85, 87, 143, 144, 412

Greece 263, 309, 331

Halicarnassus (Western Turkey)

Mausoleum 107, 108

Hamburg (Germany)

Landeskunstschule 181, 304,

Hartenbos (Western Cape) 37, 72, 74

Museum $404 \mathrm{n} 1160$

Hawick (Natal)

Mandela memorial 416

Heidelberg (Transvaal) 67 n 295

Highveld (Transvaal area) 19, 22, 358, 360, 386

Hoeko (Eastern Cape) 74

India 23, 46

Irene (Transvaal) 155

Istanbul (Turkey)

Hagia Sophia 411

Italeni (eGabeni) (Natal) 23, 24, 137, 206, 296, 371, $372,375,436$

Italy $13,59,69,91,107,116$ n 465, 132 n 522, 178, $182,194,229,245,249,251,252,258-269$,

274, 277 n 934, 304, 321, 336,

401

Johannesburg (Transvaal) 6, 82 n 363, 91 n 403, 91 n 404, 108, 109 n 450, 116, 182, 183, 268, 283 n 945 , 400 n 1144, 411, 433, 434, 458

Anglo American Corporation building 181, 182

Art Gallery 155

Cenotaph 414

City Hall 34

Empire exhibition 59, 63, 93 n 413, 106 n 438, 130, 146

John Vorster police headquarters 407

Library 69 n 300, 157, 182

Magistrates Court (Mandela statue) 416

Michael Stevenson galleries 458

Museum Africa (Africana Museum) 5, $28 \mathrm{n} \mathrm{82,54}$

n 237, 154, 155 n 600, 157, 158, 160, 161, 162, 163,
164 n 615, 166, 167, 168, 169, 291 n 779, 285, 434 n 1253, 447

Railway Station 107, 110, 117, 125 n 491, 132, 144

Rand Regiments Monument 414

Robinson Deep Gold Mine 82

Witwatersrand Technical College (Art School) $93 \mathrm{n}$ 406, 183, 186, 306

University of the Witwatersrand 409,410

Kakamas (Northern Cape)

Transformer building 86,88

Kapain (near Silkaatskop, Northern Transvaal area) 22, 23, 197 n 735, 199, 200, 201, 295, 360, 361

Karoo 74, 439

Kerkenberg/Blydevooruitsig (OFS) 61, 62, 74, 166, 296, 346, 362, 364

Retiefklip 74

Kew (UK) National Archives 6

Kimberley (Northern Cape) 27, 74, 111 Danie Theron Combat School 407, 408

King William's Town (Eastern Cape) Steve Biko Foundation and Centre 414 Steve Biko Garden of Remembrance 413 n 1191, 414

Knysna (Western Cape) 74, 428

Krugersdorp (Transvaal) Monument High School 145, 146, 153

Ladybrand (OFS) 53

Ladysmith (Natal) $51 \mathrm{n} 210,62,63$

Leipzig (Germany) Völkerschlachtdenkmal 131, 132-134

Lichtenburg (Transvaal) High School 186

Limpopo River 22, 355

Livorno (Italy) 258

London (UK) 18 n 22, 19, 116 n 465, 223

Architectural Association School of Architecture 81 British Museum 107, 136, 137, 146 Imperial College 181 Imperial War Museum 83, 84 n 372 Regent Street Polytechnic School 155 Royal College of Art 110 n 453, 111 Royal Institute of British Architects 82 South Africa House 126, 127

Victoria and Albert Museum 137 n 534, 242

Lubombo Mountains 384

Lüderitz (Namibia) 53

Luxor (Egypt)

Karnak temple $85,87,88$

Pyramid 83

Magdeburg (Germany) 181

Maqongqo Hills (Natal) 384

Marico River 360

Melbourne (Australia)

Shrine of Remembrance 132 n 523 ,

133 n 529

Middelburg (Eastern Cape) 75 
Middelburg (Transvaal) Anglo-Boer War Memorial 184

Milan (Italy) Palazzo di Giustizia 263, 333, 334

Modder River 22

Monte Altissimo (Italy) 259, 345

Moorreesburg (Western Cape) 74

Mosega (Northern Transvaal area) 22, 23, 122, $129 \mathrm{n}$ 513, 200, 360, 366

Mossel Bay (Cape) 72, 111

Munich (Germany) 178, 304, 305, 306 Akademie der Bildenden Künste 185

Natal 6, 7, 18 n 20, 19, 23, 24, 25, 28, 31 n 94, 45, 51, 53 n 223, 60, 61, 63, 67 n 293, 74, 109 n 451, 120, 123, 124, 125 n 492, 129 n 513, 152 n 581, 169, 174, 202, 203, 208, 228 n 804, 294, 296, 297, 298, 300, $360,362,363,365,366,367,369,374,375,378$, 380, 382, 383, 385, 436 n 1257, 443, 448

Natalia (Boer Republic) 25, 203, 380, 384, 38,

Ncome River (see also Blood River) 24, 378

Netherlands 17 n 19, 32, 37, 38 n 147, 47, 48, 80, 81, 124

Nylstroom (Modimolle) (Transvaal) 81, 407

Ohrigstaad (Transvaal) 61

Orange Free State/Oranje Vrystaat (OFS/OVS) 25, 27, 28, 29, 33 n 110, 53 n 223, 56, 60, 61, 63, 72, 124, $125,184,361,377,388,405$

Orange River 22, 25, 88, 125, 387

Paardekraal (Transvaal) 28, 29

Paarl (Western Cape) 438

Taalmonument 451

Paris (France) $38 \mathrm{n} 142,44,333$ Les Invalides 132 n 146

Parys (Transvaal) 72, 120

Pergamon (Western Turkey) 107, 109, 133, 242

Phongolo River 384

Pietermaritzburg (Natal) 6, 25, 41, 44, 45, 50, 51 n 210, 61, 62, 63, 74, 119 n 474, 122, 124, 125 n 493, 367, $369,375,380,381,382,385,386,405$

Church of the Vow 44, 54, 62 n 277, 122, 125 n 492, 207, 245 n 829, 287, 299, 368, 378, 380, 381, 382, 411, 438, 439

Piet Retief sculpture 119 n 474, 405, 413 n 1189 uMsunduzi Museum and Voortrekker Complex 6, 43, 368

Voortrekker Museum 43, 62 n 277, 125, 288, 297, 380, 436

Port Elizabeth (Eastern Cape) 74, 414 n 1193 Karel Landman Memorial 146 n 570

Port Natal (see also Durban) 23, 120, 122, 124, $170 \mathrm{n}$ 630, 258, 268, 275 n 933, 362, 367, 374, 376, 385

Potchefstroom (Transvaal) 61, 63, 124, 167 University of Potchefstroom 74, $170 \mathrm{n}$ 632, 444

Pretoria (Transvaal) 5, 7, 38, 41 n 165, 43, 48, 60-65, $67,72,74,75,82,83$ n 363, 122, 158 n 605, 172 n 637, 173, 194, 221, 226, 227, 234 n 810, 240, 258, 266 n 898, 268, 283 n 945, 285 n 952, 397, 400,
$401,405,407,411,420,422,423,426,433,460$,

461

City Hall 125 n 491, 126, 127, 128, 12

Ditsong National Museum of Cultural History

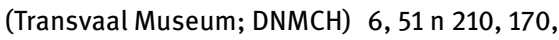
171 n 636, 216

Fort Schanskop 407, 408, 424, 426, 427, 428

Freedom Park 409, 411, 412, 413, 414, 415, 424,

429, 430

German School 181

Groenkloof Nature Reserve

Long Walk to Freedom 413, 415, 416

Harmony Hall (Deutsche Turn Halle) 60, 106, 176, 177, 178, 179 n 654, 181, 182, 183, 188-195, 198, 203, 208, 213, 216, 217, 218, 2119, 224, 225, 228, 229, 232 n 807, 233, 234, 235, 236, 239, 241, 243, 247, 248, 249, 258, 259, 268, 275, 287, 291, 293 . $317,329,400$

Heritage Centre, VTM 183 n 678, 407, 408, 426, 427, 434 n 1255

Joubert foundry 413

Kirkness contractors 228 n 804

Kruger Monument 80, 92, 93, 94 n 414, 97, 131, 413 n 1188, 461

Libertas 117

Merensky Library 132, 146, 147

Monumentkoppie 64, 65 n 288, 142, 400,

401

National Archives and Records Service of South

Africa (NARSSA) 5, 48, 80 n 348

National Film Archive 137 n 537, 139 n 539,

Nederduitsch Herformde Kerk in Afrika Argief

(NHKA) 6, 139

Police Museum 407

Portuguese Embassy 228

Pretoria College (Pretoria Boys High School) 81, 181

Pretoria Iron and Steel Works 234

Public Works Department 81, 82, 116, 264

n 882

Railway Station 93, 273

Road of Reconciliation 411, 424

Salvokop 412

South African Defence Force Memorial 430

Teachers' Training College 221

Union Buildings 59, 60, $407 \mathrm{n}$ 1174, 412

Louis Botha statue $91 \mathrm{n} \mathrm{403,} 234 \mathrm{n} 810$,

261 n 867, 416, 418

Mandela statue 416,417

University of Pretoria (Transvaal University

College) 5 n 1, 6, 19 n 29, 47, 48, 51 n 212, 72, 75 n

334, 98 n 443, 107 n 448, 108, 117, 132, 146, 147,

160 n 611, 182 n 674, 186, 268 , 291 n 969, 298,

308, 404 n 1160

University of South Africa (Unisa) 7

Vignali foundry 91, 93, 106, 107 n 440, 185, 261,

262 n 869, 400, 413, 418

Voortrekker Memorial Hall (Gedenksaal) 87, 88, 126

YSCOR/ISCOR 33 
Querceta quarry (Italy) 259, 264, 267, 268, 345

Rand/Witwatersrand (Transvaal) 27, 74, 111, 181 n 668, 183

Rensburgkoppie (Natal) 297, 372, 373

Retiefklip, see Kerkenberg

Riversdal (Riversdale, Western Cape) 74

Rome (Italy) 91 n 402, 91 n 404, 93, 116, 117, 119, 261, 268, 309 n 1022, 333, 363, 389

British Academy of Arts 131

British School at Rome 81

Canova studio 82

Trajan's Column 137, 242, 291 n 968, 389, 390, 391

Saailaer (Natal) 56, 160 n 610

Sand River (OFS) 127, 150, 202, 228, 288, 294, 371, 386, 389

Schoemansdal (Transvaal) 122

Seravezza (Italy) 259

Sharpeville (Transvaal)

Human Rights Precinct and Exhibition Centre 414

South America 97, 262

Southampton (UK)

Central School of Arts 170

South West Africa (Namibia) 53, 54

Soutpansberg (Transvaal) 22, 56, 120, 123, 148 , 158 n 606, 164, 172 n 638, 206, 224, 294, 355, 357

Soviet Union 405

Soweto

Hektor Pieterson Memorial and Museum 414, 415

Standerton (Transvaal) Concentration camp 81, 117

Stellenbosch (Western Cape) 33 n 110 University of Stellenbosch 7, 33 n 110, 36, 51, 56, 57,141

Swartberg Mountains (Eastern Cape) 74

Sydney (Australia) ANZAC memorial 131, 132, 133, 135

Syracuse (Italy)

Monument for the Legionary 33

Talanakop (Natal) 49

Thaba Nchu (OFS) 22, 23, 61, 62, 295, 359, 362
Transvaal 27, 28, 36, 47, 49, 56, 57, 60, 61, 63, $66 \mathrm{n}$ 291, 67 n 295, 72, 74, 120, 122, 124, 127 n 503, 200, $357,375,386,439$

Tugela (Thukela) River 23, 362, 367, 375, 376, 382, 383

Uganda 402

uMgungundlovu (Zululand/Natal) 23, 24, 245, 296 $299,320,362,366,367,368,379,428$ n 1237, 439 kwaMatiwane 137, 296, 320, 321, 323, 367, 368 , 371, 375

Memorial to Retief and his men 41, 42

Umzimvubu River 367

Vaal River 19, 22, 25, 63 n 278, 203, 295, 360, 387

Vegkop (OFS) 22, 23, 56, 61, 62, 74, 120, 123, 150, 152, 157, 170 n 630, 200, 206, 295, 298, 299 n 982, $326,357,358,360,362$ Vegkop Monument 436, 437, 438

Venice (Italy) 2016 Architectural Biennale 461

Ventersdorp (Transvaal) 146 n 569

Vereeniging (Transvaal) 28, 31 n 93, 31 n 94, 94 Peace Monument (Vredesmonument) 89 n 390, $110 \mathrm{n} 453$

Vryheid (Natal) 44

Wakkerstroom (Transvaal) 223

Wasbank (Natal) 74, 125 n 492

Wasbank River 376, 383

Washington (USA) Lincoln Memorial 132 Smithsonian Institution 181 n 667

Waterberg Massif 81

Weenen (Natal) 24, 61, 62

Winburg (Transvaal) 23, 50, 61, 62, 63, 71, 74, 120, 148, 152 n 581, 359

Voortrekkermonument 119 n 474, 405

Witwatersrand (Transvaal) see Rand

Worcester (Western Cape) 438

Worpswede (Germany) 304

Zambezi River/Victoria Falls 120, 122

Zuid-Afrikaansche Republiek (ZAR) 25, 27, 28, 33 n 112, $41 \mathrm{n} 165,48,63,75,93,128,294,357,358,361$, $387,389,413$ n 1188, 461 